\title{
Bacteriocin expression in sessile and planktonic populations of Escherichia coli
}

\author{
Hadeel Majeed, Lusine Ghazaryan, Moshe Herzberg and Osnat Gillor \\ The Journal of Antibiotics (2015) 68, 52-55; doi:10.1038/ja.2014.84; published online 2 July 2014
}

\section{INTRODUCTION}

When competing for nutrients and space, most bacterial species produce bacteriocins, which are ribosomally synthesized peptides with antibacterial activity. In fact, dozens of bacteriocins have been found within a single bacterial species, ${ }^{1}$ leading researchers to suggest that they have a critical role in mediating microbial interactions and maintaining microbial diversity. ${ }^{2-4}$

Colicins-bacteriocins produced by Escherichia coli-have been studied for almost a century and are a model system for bacteriocins produced by Gram-negative bacteria. ${ }^{5}$ Most colicins are encoded on operons regulated by stress, specifically DNA damage, ${ }^{5}$ and are comprised of three genes: a toxin gene encoding the colicin protein, an immunity gene encoding a protein that protects the cell from its produced toxin and a lysis gene encoding a protein that lyses the producing cell to release the colicin. ${ }^{5}$ Due to the lethality of colicin expression, the clonal colicinogenic population exhibits heterogeneity such that only a small subset of cells produces and releases colicins through lysis. This phenotypic heterogeneity has been reported in a number of colicinogenic populations with only $0.5-3 \%$ of the cells expressing colicins. ${ }^{6-8}$ All the cells within these populations express the immunity protein, regulated by a constitutive promoter, thus protected from the colicin. This behavior, carried by a handful of cells within a given population, might be explained by potential benefit to clonal siblings; by killing their adversaries, nutrients and space are thereby freed for their use.

Colicins have been identified in up to $50 \%$ of E. coli species isolated from mammals' gastrointestinal (GI) tract. ${ }^{5}$ In the GI tract, bacteria adhere to the colon epithelial cells and form a stable biofilm. ${ }^{9}$ Hence, in their natural environment, colicinogenic E. coli cells reside in a spatially structured environment in which cell-cell interactions are localized. ${ }^{10}$ Nevertheless, most studies of colicin function, expression, ecology and evolution have been performed in well-mixed environments in which the cells are free swimming. ${ }^{5}$

Many studies have compared transcription or translation profiles in planktonic versus biofilm cultures of an E. coli strain, showing differential expression of a large number of genes. ${ }^{9}$ A geneexpression study in E. coli strain K-12 showed that $5-10 \%$ of the genome is differentially expressed in these two types of culture and that genes induced in biofilm settings relate to stress responses, energy production and envelope biogenesis. ${ }^{9}$ These differences in expression profiles may also affect bacteriocin production.

Studies describing bacteriocin-mediated interactions all report a disparity in competition outcome when bacteria are sessile rather than planktonic. ${ }^{3,4,11}$ This disparity was mostly attributed to changes in the type of cell-cell interactions, from local in a spatially structured environment to global in a well-mixed environment. ${ }^{3}$ Here we suggest that the reported disparities may result not only from changes in the cells' interactions but also from differential bacteriocin expression. We present a first attempt at comparing colicin expression in biofilm and planktonic environments. To that end, we tagged a colicinogenic E. coli strain with green fluorescent protein (GFP) to monitor colicin expression in real time at the single-cell level in planktonic and biofilm settings. We predicted that in a spatially structured environment, the population would increase its bacteriocin expression, corresponding to an elevation in genes reported to be associated with stress response in E. coli biofilm. ${ }^{9,12}$

\section{MATERIALS AND METHODS}

\section{Bacterial strains and plasmids}

All experiments were performed with E. coli strain BZB1011. ${ }^{13}$ Two plasmids, pSC $303^{6}$ and pBR-ColE7 (colicin E7 operon fused to pBR322; see below), were purified using the AccuPrep Plasmid Extraction Kit (BioNeer, Seoul, South Korea) and transformed into E. coli strain BZB1011. ${ }^{13}$ Transformants were selected on the basis of antibiotic resistance and confirmed by sequencing.

\section{Plasmid construction}

Colicin E7 immunity protein was shown to mediate a site-specific cleavage of its own mRNA inhibiting the colicin and lysis activities when induction was limited. ${ }^{14}$ Due to the autoinducing nature of colicins, we monitored expression in cells carrying the colicin operon and reporter vehicles. To that end, we ligated the colicin operon (including the promoter site) to pBR322 and formed pBR-ColE7 plasmid. In addition, colicin E7 promoter was ligated to $g f p-m u t 3-$ encoding gene ${ }^{6}$ to form pSC303. Both plasmids were transferred into E. coli strain BZB1011 13 to form strain ppColE7-GFP.

\section{Growth conditions}

M9 minimal medium (Sigma, St Louis, MO, USA) and Luria-Bertani (LB) broth (Difco, Lawrence, KS, USA) were prepared according to the 
manufacturer's instructions. M9 was supplemented with $4 \mathrm{gl}^{-1}$ Casein Digest (Difco). Both media were supplemented with ampicillin at $100 \mathrm{mgl}^{-1}$ and kanamycin at $50 \mathrm{gl}^{-1}$ (Sigma) as required. Suspended cultures were grown at $25^{\circ} \mathrm{C}$ with shaking at 200 r.p.m. Biofilm cultures were grown in the flow cell (Figure 1 ) at $25^{\circ} \mathrm{C}$.

\section{Planktonic assays}

Planktonic cultures were assayed as previously described. ${ }^{8}$ Briefly, the reporter strain ppColE7-gfp was grown overnight in LB broth, diluted 1:100 and reinoculated in fresh M9 medium. Refreshed samples were collected in exponential $\left(\mathrm{OD}_{600}\right.$ of $\left.\sim 0.09\right)$, post-exponential $\left(\mathrm{OD}_{600}\right.$ of $\left.\sim 0.2\right)$ and stationary $\left(\mathrm{OD}_{600}\right.$ of $\left.\sim 0.4\right)$ growth phases. Samples were treated with $400 \mu \mathrm{g} \mathrm{ml}^{-1}$ chloramphenicol (Sigma) to block further protein synthesis and prevent lysis, and stored at $4{ }^{\circ} \mathrm{C}$ for next-day microscopy such that all samples were viewed simultaneously. The incubated cells were attached to glass slides coated with $0.1 \%$ (wt/vol) poly-L-lysine (Sigma) and fluorescence in a single cell was detected $(505-550 \mathrm{~nm})$ using laser scanning confocal microscopy (LSCM; Zeiss-Meta 510 inverted microscope, Zeiss, Oberkochen, Germany). The microscopic photos were enumerated with cellC software (http:// www.cs.tut.fi/sgn/csb/cellc/).

\section{Flow chamber construction for biofilm assays}

To monitor colicin expression at the single-cell level in a continuously growing biofilm, we designed and constructed a flow cell device (Figure 1). Multiple slides were placed in a polypropylene tub providing homogeneous growth conditions, which would otherwise only be possible in multichannel flow cells. Cover slides (Marienfeld, Lauda-Königshofen, Germany) were placed in the grooves for biofilm cultivation. Slides were removed and replaced by sterile ones, such that the media flow remained unchanged.

\section{Biofilm assays}

As substratum for biofilm growth, hydrophilic polypropylene filters (Millipore, Billerica, MA, USA) were attached to glass slides using thin, inert doubleadhesive tape (Nitto Denko, Osaka, Japan). The polypropylene substrate did not cause autofluorescent emission during microscopic analyses. The flow cell (Figure 1), slides and associated silicon tubing were autoclave sterilized and the polypropylene substrates were disinfected with $70 \%$ ethanol. Thereafter, the flow cell was conditioned by injecting it with Casein Digest-supplemented M9 medium for $3 \mathrm{~h}$. Biofilm inoculum was prepared using the reporter strain, ppColE7-gfp, grown overnight in LB medium, reinoculated in fresh M9 medium at 1:100 dilution and grown to early exponential phase $\left(\mathrm{OD}_{600}\right.$ of $\sim 0.07)$. The culture $(150 \mathrm{ml})$ was then injected into the flow channel after medium flow was arrested. The flow of M9 medium without bacteria was resumed $1 \mathrm{~h}$ after the inoculation step at a constant rate of $2 \mathrm{ml} \mathrm{min}^{-1}$ using a peristaltic pump (MasterFlex 07524-40, MasterFlex, Verenon Hills, IL, USA). Biofilm cultures were monitored 24, 48 and $72 \mathrm{~h}$ after cell inoculation using LSCM.

Unlike planktonic cells that are easily discerned, biofilm cells are hard to tell apart and thus were stained with $300 \mathrm{~nm}$ 4',6-diamidino-2-phenylindole (DAPI; Sigma), a nucleic acid stain that preferentially stains double-stranded DNA, whereas the cells expressing colicins were marked with GFP. DAPI was diluted in phosphate buffer saline (PBS; Sigma), applied to the removed slide for $2 \mathrm{~min}$ and then rinsed three times in PBS.

Microscopic observation and image acquisition were performed using LSCM at $\times 63$ magnification, equipped with detectors and filter sets for monitoring DAPI-stained cells $(420-480 \mathrm{~nm})$ and GFP expression. A minimum of 30 spots per sample were tested. As cells are hard to detect in biofilm images, the percentage of colicin producers was estimated as the percentage of GFP from the total blue (DAPI) and by biovolume estimations. Green-blue scale images were measured using ImageJ (http://rsb.info.nih.gov/ij/ index.html). In addition, the biovolumes $\left(\mu \mathrm{m}^{2} \mu \mathrm{m}^{-3}\right)$ of the biofilms were calculated using IMARIS software v7.1.1 (Bitplane, Zurich, Switzerland). Three-dimensional reconstruction of the LSCM images taken from the biofilms was carried out using IMARIS software.

\section{RESULTS AND DISCUSSION}

We investigated the heterogeneity of bacteriocin expression in clonal populations of biofilm and planktonic E. coli. Several studies have described the phenotypic heterogeneity in planktonic colicinogenic populations, with colicins being expressed by a small subset $(0.5-3 \%)$ of the colicinogenic population. ${ }^{6-8}$ Cells simultaneously produced the colicin toxins and reported their expression without impairing their fitness. The generation time, $\mu$, (calculated as doubling time per hour) in minimal medium of the strain harboring only the colicin operon, pBR-ColE7, was similar to that of the colicin reporter strain, pSC303 ( $\mu=0.28 \pm 0.04$ and $0.25 \pm 0.05$, respectively). This was probably due to the low copy numbers of the colicin-producer (15-20 copies) ${ }^{15}$ and -reporter $(6 \text { copies })^{13}$ plasmids.

We evaluated the percentage of colicin-producing cells in a culture of planktonic E. coli strain ppColE7-GFP to find that during exponential growth phase, $2.3 \pm 1.2 \%$ of the bacterial population emitted fluorescence (Figure 2a), whereas in the post-exponential and stationary phases, $1.8 \pm 0.2$ and $1.4 \pm 0.2 \%$ of the respective cells fluoresced (Figures 2a and c). E. coli strain BZB1011 harboring pSC303 alone emitted similar levels of fluorescence $(1.5 \pm 0.3 \%)$, suggesting that the colicin operon does not impact the reporter

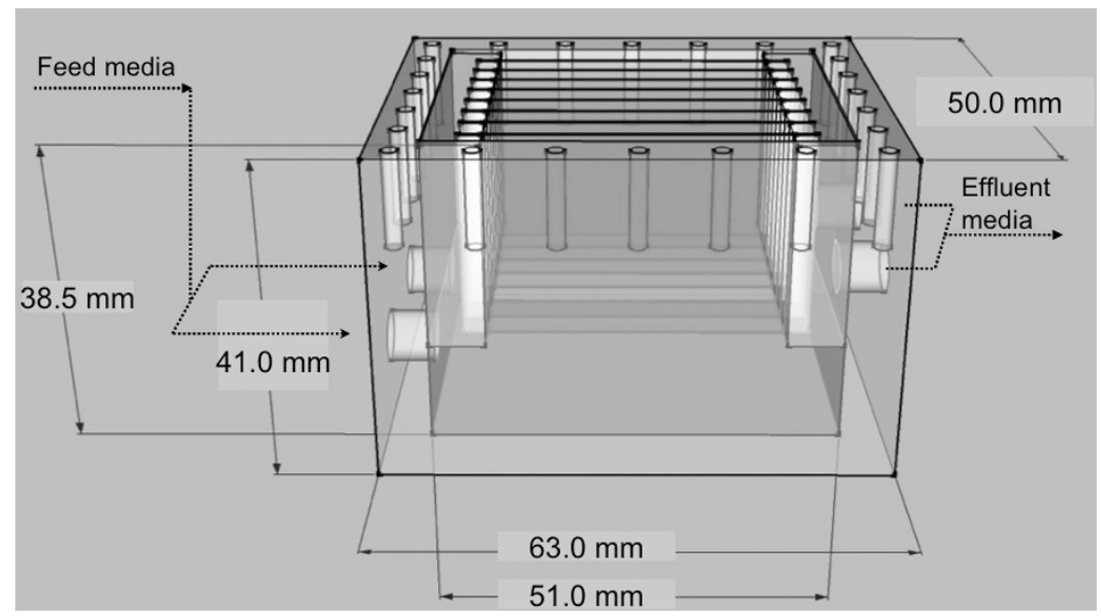

Figure 1 Multiple surface flow cell device. The flow cell contains eight grooves that enable sampling by removal and replacement of the $40 \times 24 \mathrm{~mm}$ coverslip slides. A full color version of this figure is available at The Journal of Antibiotics journal online. 


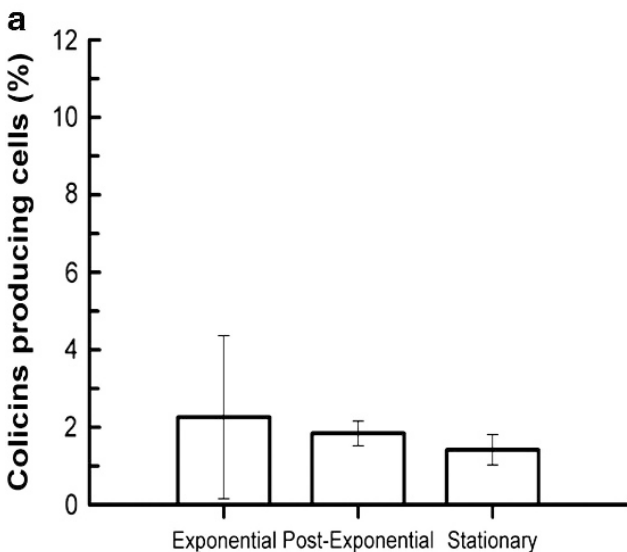

Growth phase

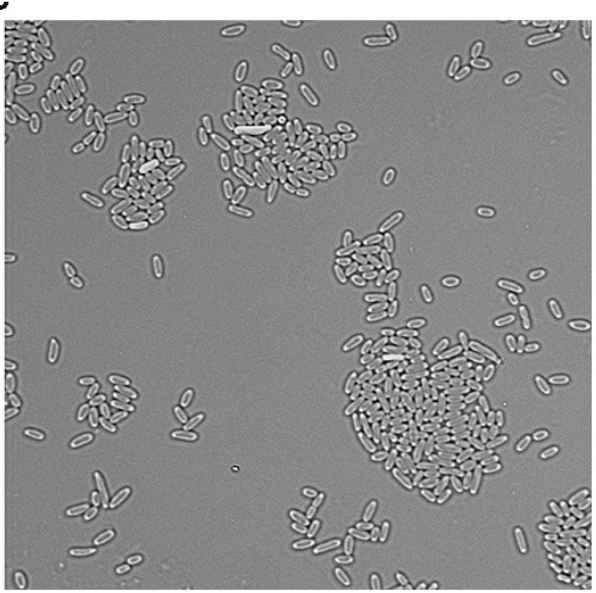

b

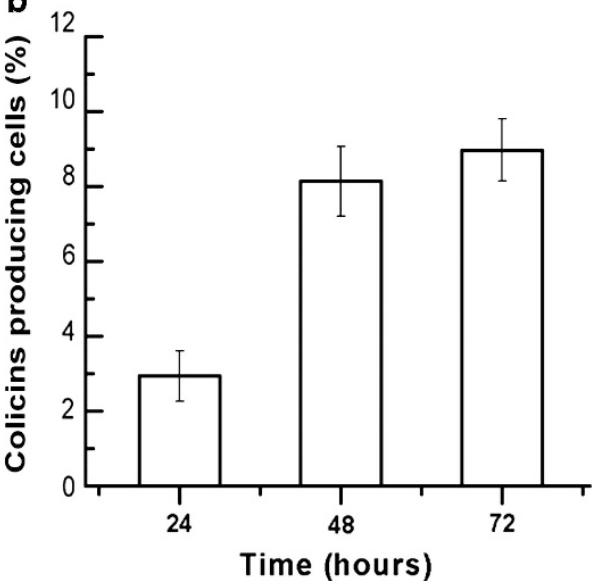

d

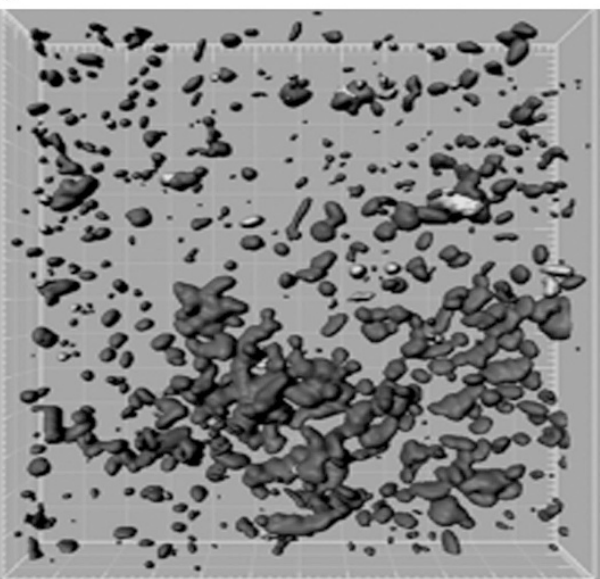

Figure 2 Colicin expression in planktonic and biofilm cultures. (a) Percentage of colicin-expressing cells (reported by green fluorescent protein (GFP) expression) in exponential, post-exponential and stationary-phase planktonic cells. Values are average and s.d. from at least three experiments where a total of 10000 cells were sampled. (b) Percentage of colicin expression in biofilm established 24,48 and $72 \mathrm{~h}$ after inoculation into the flow cell. Values are average and s.d. from at least three experiments where the percentage was calculated from the biovolumes of GFP to DAPI-stained cells. Confocal laser microscopic image of E. coli-expressing cells' GFP in (c) stationary-phase free-swimming cultures and in (d) biofilm culture grown for $72 \mathrm{~h}$ (DAPI-stained E. coli cells). A full color version of this figure is available at The Journal of Antibiotics journal online.

expression (data not shown). This follows previous observations of colicins A, E1, E7, $\mathrm{K}$ and $\mathrm{N}$ being produced by a small fraction $(0.5-$ $3 \%)$ of their respective E. coli populations during stationary growth phase $^{6-8}$ The heterogeneity within the clonal population was attributed to low stochastic stress response in a given population, which triggers colicin expression. ${ }^{6-8}$ It may be postulated that this type of stochasticity, together with the self-destructive nature of colicin, is predicted to engage a small fraction of the population.

To test ColE7-expressing cells in a biofilm setting, we designed and constructed a flow cell (Figure 1) that enables detection of gene expression at specific time points in a continuously growing biofilm. The performance of the flow cell was tested and found to support a similar biofilm mass in each run, as similar biovolumes were measured at the attached polypropylene filter after $24 \mathrm{~h}$ of biofilm growth (data not shown).

The intervals tested, the percentage of colicin-expressing (i.e., fluorescent) cells in the biofilm accounted for $2.9 \pm 0.6,8.1 \pm 0.9$ and $8.9 \pm 0.8 \%$ (Figure $2 \mathrm{~b}$ and $\mathrm{d}$ ). Biovolumes for total cells and those expressing colicins at $72 \mathrm{~h}$ were $0.3 \pm 0.16$ and $0.025 \pm 0.02 \mu \mathrm{m}^{3}$, respectively ( $n=5$ analyzed areas). Our results suggest that colicin expression in a biofilm setting is two- to three- folds higher than stationary-phase planktonic cultures (Figure 2). This observation counters the dogma in modeling bacteriocin interactions claiming that bacteriocins are secreted at a constant rate proportional to their growth. ${ }^{16,17}$ Contrastingly, our results suggest that changing environmental settings alter bacteriocins' production.

Stress-related genes were found to be upregulated in biofilm settings. For instance, genes regulated by the SOS system (involved in repairing DNA damage), such as $\operatorname{rec} A$, $\operatorname{din} I$ and $\operatorname{sul} A$, were upregulated by twofold or more in E. coli biofilms compared with their respective expression in stationary-phase planktonic cultures. ${ }^{9,12}$ Concomitantly, the SOS system regulates colicin expression, ${ }^{6,7}$ which may account for the reported increase in colicin-producing cells in sessile compared with that in free-swimming cultures (Figure 2). Similarly, only sessile cultures of the oral bacterium Streptococcus mutans grown in a biofilm or in large aggregates produced bacteriocins, whereas no production was detected in planktonic cultures. ${ }^{18}$

The outcome of bacteriocin-mediated interactions in spatially structured environments such as the surface of a petri plate $3,4,11$ or mouse GI tract ${ }^{4}$ has little in common with the results obtained when the same populations interact in suspension. When cells are sessile and not free swimming, lower concentrations of bacteriocin producers 
are required to invade and outcompete bacteriocin-sensitive populations. ${ }^{3,19}$ Similarly, sessile bacteriocin producers persist for prolonged periods in a quasi-stable coexistence, whereas competition in a well-mixed, unstructured environment is rapidly resolved. ${ }^{3,4,11}$ In both cases, elevated colicin production in spatially structured environments may shift the balance such that the colicinogenic cells can successfully invade susceptible populations (even when initially sparse) or defend their habitat against invaders. This is of particular relevance to the control of biofilm-forming pathogens, a major objective to researchers and medical professionals alike. ${ }^{17,20}$ Here we provide indication to the potential use of bacteriocins in biofilm settings as the increase in their production could be applied to target residing or invading pathogens. Moreover, bacteriocin's role in biofilms may extend beyond competition or defense, as it has been suggested to include quorum-sensing mediators ${ }^{10}$ and communication signals. ${ }^{20}$ These roles may be facilitated by the enhanced production we demonstrated here and follow the prediction of various models 2,16 claiming that bacteriocins are most advantageous in a spatially structured environment where interactions are local.

\section{ACKNOWLEDGEMENTS}

This work was supported by National Institutes of Health grants R01A1064588-01A2 to OG. HM is a recipient of a Ph.D. grant from the Israel Council of Higher Education and of a scholarship from the Israeli Ministry for Science and Technology for Women in Science Grant 3-6485.

1 Riley, M. A. \& Wertz, J. E. Bacteriocins: evolution, ecology, and application. Annu. Rev. Microbiol. 56, 117-137 (2002).

2 Inglis, R. F., Gardner, A., Cornelis, P. \& Buckling, A. Spite and virulence in the bacterium Pseudomonas aeruginosa. Proc. Natl Acad. Sci. USA 106, 5703-5707 (2009).
3 Kerr, B., Riley, M., Feldman, M. \& Bohannan, B. Local dispersal promotes biodiversity in a real-life game of rock-paper-scissors. Nature 418, 171-174 (2002).

4 Majeed, H., Gillor, O., Kerr, B. \& Riley, M. A. Competitive interactions in Escherichia coli populations: the role of bacteriocins. ISME J. 5, 71-81 (2011).

5 Cascales, E. et al. Colicin biology. Microbiol. Mol. Biol. Rev. 71, 158-229 (2007).

6 Kamensek, S., Podlesek, Z., Gillor, O. \& Zgur-Bertok, D. Genes regulated by the Escherichia coli SOS repressor LexA exhibit heterogenous expression. BMC Microbiol. 10, 283 (2010).

7 Mrak, P., Podlesek, Z., van Putten, J. \& Žgur-Bertok, D. Heterogeneity in expression of the Escherichia coli colicin $\mathrm{K}$ activity gene cka is controlled by the SOS system and stochastic factors. Mol. Genet. Genomics 277, 391-401 (2007).

$8 \mathrm{Mulec}$, J. et al. A cka-gfp transcriptional fusion reveals that the colicin $\mathrm{K}$ activity gene is induced in only 3 percent of the population. J. Bacteriol. 185, 654-659 (2003).

9 Beloin, C., Roux, A. \& Ghigo, J. M. Escherichia coli biofilms. Curr. Top. Microbiol. Immunol. 322, 249-289 (2008).

10 Von Bodman, S. B., Willey, J. M. \& Diggle, S. P. Cell-cell communication in bacteria: united we stand. J. Bacteriol. 190, 4377-4391 (2008)

11 Majeed, H., Lampert, A., Ghazaryan, L. \& Gillor, O. The weak shall inherit: bacteriocin-mediated interactions in bacterial populations. PLOS ONE 8, e63837 (2013).

12 Beloin, C. \& Ghigo, J. M. Finding gene-expression patterns in bacterial biofilms. Trends Microbiol. 13, 16-19 (2005).

13 Pugsley, A. Escherichia coli K12 strains for use in the identification and characterization of colicins. J. Gen. Microbiol. 131, 369-376 (1985).

14 Hsieh, S.-Y. et al. A novel role of ImmE7 in the autoregulatory expression of the ColE7 operon and identification of possible RNase active sites in the crystal structure of dimeric ImmE7. EMBO J. 16, 1444-1454 (1997).

15 Zaslaver, A. et al. A comprehensive library of fluorescent transcriptional reporters for Escherichia coli. Nat. Methods 3, 623-628 (2006)

16 Frank, S. A. Spatial polymorphism of bacteriocins and other allelopathic traits. Evol. Ecol. 8, 369-386 (1994)

17 Bucci, V., Nadell, C. D. \& Xavier, J. B. The evolution of bacteriocin production in bacterial biofilms. Am. Nat. 178, E162-E173 (2011).

18 Qi, F., Chen, P. \& Caufield, P. W. Purification and biochemical characterization of mutacin I from the group I strain of Streptococcus mutans, $\mathrm{CH} 43$, and genetic analysis of mutacin I biosynthesis genes. Appl. Environ. Microbiol. 66, 3221-3229 (2000).

19 Chao, L. \& Levin, B. R. Structured habitats and the evolution of anticompetitor toxins in bacteria. Proc. Natl Acad. Sci. USA 78, 6324-6328 (1981).

20 Hibbing, M., Fuqua, C., Parsek, M. \& Peterson, S. Bacterial competition: surviving and thriving in the microbial jungle. Nat. Rev. Microbiol. 8, 15-25 (2010). 